\title{
Ultra-Widefield Fluorescein Angiographic Patterns, Retinal Microvascular Anomalies and Retinal Ischemic Index in Branch Retinal Vein Occlusions with Established Retinal Neovascularization
}

This article was published in the following Dove Press journal: Clinical Ophthalmology

Peck Lin Lip $\mathbb{D}$

Hemalatha Kolli

Dipti Trivedi

Birmingham and Midland Eye Centre, Birmingham, UK
Correspondence: Peck Lin Lip Birmingham and Midland Eye Centre Sandwell \& West Birmingham NHS Trust City Hospital, Dudley Road, Birmingham BI8 7QH, UK

Tel +44 I2I 554380 I

$\mathrm{Fax}+44$ |2| 507679 |

Email pllipwoo@gmail.com
Purpose: To share the experience of using ultra-widefield fluorescein angiography (UWFFA) in recognizing the potential signs for retinal neovascularizations (NVE) development in branch retinal vein occlusions (BRVO).

Methods: Reporting angiographic findings in 60 BRVO eyes presenting with NVE and vitreous hemorrhage using UWF-FA investigation. Angiographic retinal ischemic index (ARI) was also calculated from UWF-FA as the ratio of digitally mapped ischemic retina area against area of optic disc, termed unit of disc diameter (DD).

Results: We observed emerging angiographic features common to these patients: pattern of a localized non-perfused retina at early phase of UWF-FA remaining non-perfused at the late phase (black retinal ischemia, black-RI) (100\%); presence of retinal microvascular anomalies (RMAs) at the "water-shed-border" of black-RI (100\%); site of NVEs observed at either the same "water-shed-border" (42\%) or from the main vessel branch within the black-RI $(30 \%)$, or from both sites (28\%); multiple NVEs were observed in all eyes except two with single active NVE. Median ARI size was 114 DD (SD 80 DD), range 5-354 DD.

Conclusion: We report a recurring angiographic pattern common to eyes with active BRVO-NVEs from UWF-FA, and NVEs in this clinical group can develop from varied ARI sizes. Further studies would be needed to establish the role of UWF-FA in predicting angiographic risk factors for BRVO-NVE.

Keywords: definition, vitreous hemorrhage, ghost vessels, risk, laser

\section{Plain Language Summary}

Clinicians are frequently faced with patients presented with sudden visual loss due to vitreous hemorrhage secondary to retinal vein occlusion (RVO). Vitreous hemorrhage happens because of the development of retinal neovascularization (NVE) which is a late complication following the initial event of ischemic RVO. Although there is plenty of published literature on the treatment and management of this complication, there is very limited information on angiographic studies focused on NVE complexity. In recent years, the advancement of angiographic imaging devices such as ultra-widefield angiography (UWF-FA) has allowed a much wider view of pathological retina and hence potential better understanding of the disease itself. We have studied UWF-FA in patients with active NVE secondary to branch retinal vein occlusion and report an interesting recurring theme of recognizable angiographic ischemic pattern common to this group of patients. Our information may enhance the understanding and the clinical management of NVE in this disease subgroup with the increasing application of UWF-FA in routine clinical practice. 


\section{Introduction}

Retinal neovascularization (NVE) is a commonly encountered late complication of branch retinal vein occlusions (BRVO). Patients who develop this late complication often present with sight-threatening symptoms of vitreous hemorrhage. In ischemic BRVO eyes, the incidence of NVE development was reported to be $22 \%$ over a 3 -year period, and $61 \%$ of untreated active NVE would have vitreous hemorrhage, compared to $29 \%$ of treated NVE. ${ }^{1}$ Although retinal ischemia and subsequent elevated ocular levels of vascular endothelial growth factor are well understood and are considered to be one of the many important factors in the pathogenesis of NVE development, very little is studied on risk stratification and angiographic retinal signs to aid better prediction of NVE development. ${ }^{2,3}$ In the Branch Retinal Vein Occlusion Study (BRVOS 1986), ischemic retina was depicted by the limited 7-field angiographic imaging, and retinal capillary non-perfusion of 5 disc diameter (DD) or more was reported to relate to $41 \%$ of NVE development with vitreous hemorrhage. ${ }^{1}$

In recent years, advancement in imaging devices such as ultra-widefield fundus fluorescein angiogram (UWF-FA) has allowed the capture of a much wider retina view from a single image and greater visualization of other peripheral retinal pathology. ${ }^{4,5}$ In addition, few studies had concentrated on re-defining the angiographic retinal ischemic index (ARI) and associating its importance with NVE development in central retinal vein occlusions (CRVO) but not in BRVO. ${ }^{6-8}$

In our daily clinical practice of interpreting UWF-FA, we repeatedly observed a recurring theme of common angiographic features in BRVO eyes with evidence of vitreous hemorrhage from active NVE. Herein, we share our observation of the UWF-FA angiographic features and ARI (size of retinal ischemia) calculation to associate with NVE development.

\section{Methods}

This was a single-center retrospective observational study on UWF-FA imaging analysis based on all BRVO inclusive of hemi-retinal vein occlusion (HRVO) patients presented to our tertiary-referral and eye emergency center. All patients in this study had vitreous hemorrhage and evidence of active NVEs, underwent routine standardized clinical care of ophthalmic assessments and investigations including UWF-FA (Optos200Tx or Optos California, Optos PLC, Dunfermline, United Kingdom).

\section{Study Idea and Patient Source}

The study idea and subsequent identification of this specific patient subgroup (BRVO/HRVO with active NVE) were primarily generated from one of the authors (PLL) routinely interpreting results of fluorescein angiogram for all RVO patients attending the virtual fluorescein angiogram clinics. There were 1-2 such clinics per month as part of the RVO clinical service in our eye center, whereby all relevant ophthalmic investigations (vision record, intraocular pressure check, optical coherence tomography and fluorescein angiography) were performed by nurses; the test results were then interpreted and reported by a senior clinician at a later stage with further reviews and treatment organized for patients according to their test results. Additional study patient sources were also obtained from electronic medical records database on subgroup specification of BRVO-vitreous hemorrhage and BRVO-NVE.

\section{Study Inclusions and Exclusions}

Study inclusion criteria were BRVO/HRVO patients with a record of vitreous hemorrhage when occurring at initial presentation or at any stage of follow-up period; patients had UWF-FA performed at least once after diagnosis confirming presence of active NVE. The study excluded patients who had poor UWF-FA images not clear enough for study analysis and mapping and patients who never had UWF-FA (due to clinical reasons or who had not presented before the time of UWF-FA availability in our eye center). Herein, the retrospective study process of recruitment and image analysis was conducted continuously over a period of 2 years from July 2017 to July 2019 ; study inclusive of UWF-FA image availability was from June 2016 to July 2019 when the Optos machine was first available in our eye center.

\section{Angiography Procedure, Data and Image Analyses}

The angiographic images were generated as a series of single-center $200^{\circ}$ widefield images using Optos machine captured during an episode of UWF-FA of a study patient. Image mapping was performed on selected first clear image with depictable area of retinal ischemia with active NVE regardless of the presence or absence of laser photocoagulation. Angiographic retinal ischemia is termed as "black retinal ischemia" (black-RI) in this study referring to the identifiable area of nonperfused retina at an early 
phase of angiogram (20 seconds to 1 minute) and remained nonperfused at the late phase (3 to 5 minutes) of angiogram (Table 1 definitions). Local UWF-FA protocol was applied to capture a series of single-center images over 5 minutes: starting from the "disease study eye" for the first angiography run of the first minute, then switching to capture the contralateral eye for the next minute, before returning to the study eye to end the angiography ending by 5 minutes. UWF-FA was performed as the initial investigation for all patients and repeated usually 4-6 monthly over the follow-up duration.

The Angiographic Retinal Ischemic Index (ARI) was defined as the angiographic ratio of black-RI area against the area of the optic disc, termed the unit of disc diameter (DD) in this study. The digital mapping of both black-RI and optic disc areas were delineated using the in-built Optos California software function of area measurement (Figure 1). The image best for mapping was usually from the early phase of angiography run of the initial 30-60 seconds. ARI mapping was done on the original definable black-RI with definable NVE. In some patients with previous history of laser treatment, mapping was done based on the residual delineated black-RI area with definable active NVE.

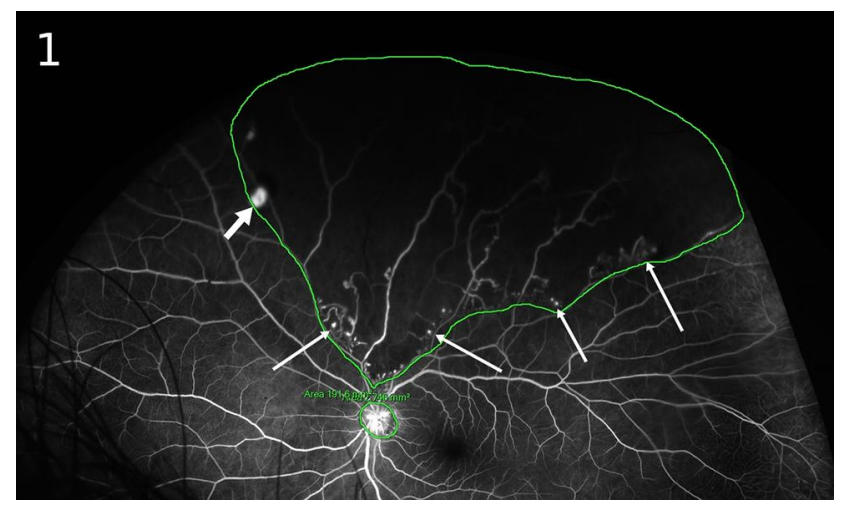

Figure I Ultra-widefield mapping on angiographic black retinal ischemia. Ultrawidefield fluorescein angiogram (UWF-FA) shows an identifiable "black retinal ischemia" (black-RI) as the nonperfused retina delineated by a water-shed-border. The digital mappings (demarcated borders) of black-RI and optic disc were generated from an Optos-California model of in-built software function, giving a calculation of Angiographic Retinal Ischemic Index (ARI) as the ratio of the black-RI area against the area of the optic disc. UWF-FA also illustrates retinal neovascularization (short arrow) and retinal microvascular anomalies (long arrows) as numerous tiny hyperfluorescent dots sharing the same water-shed border of black-RI.

Records of patients' demographics, presenting symptoms, past ocular and treatment histories, presence of vitreous or pre-retinal hemorrhages were retrospectively obtained from electronic medical notes. The analysis of UWF-FA images included these characteristics: active

Table I Definitions Used in This Study

\begin{tabular}{|c|c|}
\hline $\begin{array}{l}\text { Black retinal ischemia } \\
\text { (black-RI) }\end{array}$ & $\begin{array}{l}\text { Refers to an identifiable area of nonperfused retina at an early phase of fluorescein angiogram (initial } 20-60 \\
\text { seconds) and remained nonperfused at the late phase of angiogram (last 3-5 minutes), indicating the presence of } \\
\text { a localised sector of ischemic retina as a result of the BRVO event. }\end{array}$ \\
\hline Water-shed-border & $\begin{array}{l}\text { Refers to an angiographically delineable border of black-RI. The border contrasts nonperfused black-RI from the } \\
\text { adjacent normal (perfused) retina, marking the territory boundary of ischemic retina corresponding to the BRVO } \\
\text { event. }\end{array}$ \\
\hline $\begin{array}{l}\text { Angiographic retinal } \\
\text { neovascularization } \\
\text { (NVE) }\end{array}$ & $\begin{array}{l}\text { Refers to an abnormal retinal vascular network which becomes hyperfluorescent at the early run of fluorescein } \\
\text { angiogram and leaks profusely (increased intensity of hyperfluorescence) at the late phase of fluorescein } \\
\text { angiogram. The activity of NVE was counterchecked with the color fundus photograph taken preceding the start } \\
\text { of angiogram, confirming corresponding red NVE network. }\end{array}$ \\
\hline $\begin{array}{l}\text { Retinal microvascular } \\
\text { anomalies (RMAs) }\end{array}$ & $\begin{array}{l}\text { RMAs are multiple benign-looking red dot-caliber of abnormal retinal vasculatures (similar clinical appearance as } \\
\text { commonly termed microaneurysms, telangiectasia or retinal collaterals), often detectable in the late "healed" } \\
\text { phase of BRVO or HRVO when the initial BRVO retinal hemorrhages have resolved. Their differentiation from } \\
\text { NVE is based on an angiographic picture of early fluorescein uptake but without increasing hyperfluorescence at } \\
\text { the late phase. Clinically visible in "BRVO territory," they are detectable angiographically and specifically at } \\
\text { ischemic "water-shed-border" in our cohort. }\end{array}$ \\
\hline Retinal ghost vessels & $\begin{array}{l}\text { These are angiographically nonperfused retinal vessels and can be seen clinically and on fundus photographs as } \\
\text { empty white sclerosed retinal vessels after the initial BRVO retinal hemorrhages have resolved, often considered } \\
\text { as an "old inactive BRVO" feature. }\end{array}$ \\
\hline $\begin{array}{l}\text { Angiographic } \\
\text { "3Bs pattern" }\end{array}$ & $\begin{array}{l}\text { Refers to an angiographic pattern illustrated by ultra-widefield imaging of three commonly recurring features seen } \\
\text { in this cohort: black-RI, water-shed ischemic border, and budding of RMAs where NVEs are also found sharing the } \\
\text { same border. }\end{array}$ \\
\hline
\end{tabular}


NVEs (locations), retinal microvascular anomalies, ghosting of retinal vessels and mapping of retinal ischemic size to determine ARI. The data collection and image analysis were performed by all three authors with all image selection approved and image mapping process trained by the senior author (PLL).

\section{Duration of NVE Development}

This was an approximate calculation based on the timing of previous/initial BRVO events on ipsilateral eye to the present episode of BRVO presentation with vitreous hemorrhage occurrence or angiographic evidence of NVE, whichever was sooner. The previous initial ipsilateral BRVO episode could be in our records if the patient had first presented to our eye center or from the patient's history alone if they had initially attended other eye hospitals. We were unable to calculate the duration of NVE development in patients who first attended us with vitreous hemorrhage but had no history or record of previous ipsilateral BRVO event.

\section{Ethics and Statistical Analysis}

This retrospective observational cohort was conducted at Birmingham \& Midland Eye Centre, Birmingham, United Kingdom with the ethical approval from the Institutional Review Board (Sandwell and West Birmingham Research and Development review board) in accordance with the "Good Clinical Practice" regulations in the United Kingdom and adhered to the tenets of the Declaration of Helsinki. Informed written consents were obtained from all patients for UWF-FA investigation plus any treatment procedures, as part of the routine and standard clinical care in our real-world clinical practice. Relevant study angiographic images and photographs were anonymized. Descriptive statistics were used to show demographics and baseline characteristics. Statistical analyses were performed using Microsoft Excel 2016 for Windows and SSPS Statistics (IBM Corp., Armonk, USA).

\section{Results}

We identified 60 BRVO eyes of 60 patients who attended our eye center with vitreous hemorrhage either as an initial presenting complaint $(35 / 60$ eyes, 58\%) or later at a review visit. Of the 60 eyes, $53(88 \%)$ had BRVO and 7 (12\%) HRVO. The study cohort included 35/60 (58\%) males; mean age of the cohort was 62 years old (standard deviation 11 years, age range from 28 to 91 years old). Although 10/60 patients (17\%) had established diabetes, none had angiographic evidence of diabetic retinopathy in the contralateral eye. All patients had a recorded history of hypertension treated or new.

\section{Estimated Duration of NVE Development}

In our series, $10 / 60$ patients (17\%) had a clear history of previous RVO episodes ranging from 1.7 years to 50 years prior to first presentation to our eye center (Table 2). The history was provided by individual patients who recalled the ipsilateral BRVO event diagnosed and treated in other hospitals. In this cohort, we had angiographic records of NVE progression or new NVE development in 12/60 eyes (18\%) over an average time scale of 22.4 months (SD 11.9, median 21 months, range 7-50 months). Estimation of duration of NVE development was not possible for patients in this cohort whose NVE and ischemia were well treated with laser photocoagulation over the course of follow-up following the initial presentation to our eye center. Table 2 provides further information on the estimated duration of NVE development of a total of 22/60 eyes $(36 \%)$ in this cohort, based on history or angiographic captured of NVE development/progression from inadequately treated eyes.

Table 2 Duration of NVE Development and Angiographic Retinal Ischemic Index

\begin{tabular}{|c|c|c|c|}
\hline \multicolumn{4}{|c|}{$\begin{array}{l}\text { Duration of NVE Development (Estimation Based on } \\
\text { History and Captured Angiographic Timing) }\end{array}$} \\
\hline$N=60$ eyes & $\begin{array}{l}\text { Elicited } \\
\text { from } \\
\text { patients' } \\
\text { history } \\
10 \text { eyes } \\
(16.7 \%)\end{array}$ & $\begin{array}{l}\text { Timing from } \\
\text { angiography } \\
\text { follow-up } \\
12 \text { eyes } \\
(20 \%)\end{array}$ & $\begin{array}{l}\text { Total number in } \\
\text { cohort with known } \\
\text { duration of NVE } \\
\text { development } \\
22 \text { eyes } \\
(36.7 \%)\end{array}$ \\
\hline Mean duration & $\begin{array}{l}13.6 \\
\text { years }\end{array}$ & 22.4 months & $\begin{array}{l}86.2 \text { months } \\
\text { ( } 7.2 \text { years) }\end{array}$ \\
\hline SD & $\begin{array}{l}15.5 \\
\text { years }\end{array}$ & 11.9 months & $\begin{array}{l}\text { I4I.3 months } \\
\text { (I I.8 years) }\end{array}$ \\
\hline Median & 8.5 years & 21 months & $\begin{array}{l}26.5 \text { months } \\
(2.2 \text { years) }\end{array}$ \\
\hline Range & $\begin{array}{l}1.7 \text { to } 50 \\
\text { years }\end{array}$ & $\begin{array}{l}7 \text { to } 50 \\
\text { months }\end{array}$ & $\begin{array}{l}7 \text { to } 600 \text { months } \\
\text { ( } 0.6 \text { to } 50 \text { years) }\end{array}$ \\
\hline \multicolumn{4}{|c|}{ Angiographic Retinal Ischemic Index (ARI) } \\
\hline $\begin{array}{l}\text { Mean (SD) } \\
\text { Median (Range) }\end{array}$ & $\begin{array}{l}114 \mathrm{DD}( \\
93 \mathrm{DD}(5\end{array}$ & DD) & \\
\hline
\end{tabular}

Abbreviations: NVE, retinal neovascularization; $\mathrm{N}$, total number of eyes studied in this cohort; SD, standard deviation; DD, disc diameter as unit for ARI. 
Prior to the angiographic diagnosis of NVE, 26/60 eyes $(43 \%)$ received some degree of laser treatments related to the RVO event (verified from fluorescein angiogram) and $23 / 60$ eyes (33\%) had received intravitreal anti-vascular endothelial growth factor (anti-VEGF) injections in this cohort. In particular, for the 12 eyes with angiographiccaptured NVEs development, 10/12 (83\%) had received laser treatment, $11 / 12$ eyes $(91.7 \%)$ had received antiVEGF injections with a mean frequency of five injections $(\mathrm{SD}=2.7$, median $=5$, range $0-9)$ prior to angiographic diagnosis of NVEs.

\section{Angiographic Retinal Ischemic Index (ARI)}

Table 2 shows the calculated mean ARI in this cohort as 114 DD (standard deviation $80 \mathrm{DD}$ ), the size of retinal ischemia ranged from $5 \mathrm{DD}$ to $354 \mathrm{DD}$ with a median of 93 DD. In this cohort, we found all the vascular occlusion sites originated from the proximal end of a major vascular branch (within the posterior pole), the corresponding black-RI territories recorded to involve posterior pole, continued into mid-peripheral and reached a wider extension in the far peripheral retina. There were 40/60 eyes (67\%) which had ischemia involving macula.

\section{Ultra-Widefield Angiographic Patterns and Features}

From the UWF-FA images, we observed three recurring angiographic features common to all eyes: 1) the presence of black-RI; 2) definable water-shed ischemic border; and 3) numerous retinal microvascular anomalies (RMAs) and NVEs located at this border (Figure 2A-D).

\section{Retinal Neovascularization (NVE)}

Angiographic NVE definition is as in Table 1. We found that all NVEs arose from within or at the border of the defined black-RI areas: $25 / 60$ eyes (42\%) were observed to have NVEs sharing the same water-shed ischemic border with RMAs; $18 / 60$ eyes (30\%) had NVEs arising from the bigger vessel-border within black-RI and 17/60 (28\%) had mixed origins of both sites (Table 3). We also observed 31/ 60 eyes (52\%) had NVE network developed within or partly in any macula defined within black-RI territory. Most eyes had developed multiple NVEs, only two eyes developed a single NVE in their respective ischemic retina, with a recorded ARI size of 5DD and 12DD.

\section{Retinal Microvascular Anomalies (RMAs)}

RMAs presence was visible clinically (as red dots on retina similar to microaneurysms, retinal telangiectasia/ collaterals) and more obviously definable in UWF-FA (Table 1 definition). They were found at the water-shed border of the entire black-RI territory in UWF-FA. Their numbers were more numerous in the posterior pole compared to the far peripheral retina as peripheral delineation is less well-captured by UWF-FA. RMAs were observed to share the same water-shed borders as some of the original sites of NVE (Table 3).

\section{Retinal Ghost Vessels}

Clinically seen as white sclerosed retinal vessels (Table 1 definition, Figure 3A), our study recorded their presence in 47/60 eyes (78\%) (Table 3). The presence of ghost vessels was recorded in all patients who had a previous history of BRVO treated or untreated, reflecting the healing late phase of an BRVO event. UWF-FA analysis recorded black-RI as a constant feature found around all ghost vessels in this cohort (Figure 3B).

\section{Discussion}

The presence of retinal ischemia has been the only well understood risk factor driving the development of retinal neovascularization in many retinovascular diseases. In our cohort of BRVO eyes with established NVE and vitreous hemorrhage, we report the association of the size and site of retinal ischemia, the potential time scale for an NVE development in eyes with untreated or under-treated retinal ischemia and other recognizable UWF-FA angiographic patterns and related angiographic features to NVE development.

\section{Angiographic Retinal Ischemic Index (ARI)}

The retinal ischemic index in ischemic CRVO has been studied with ARI of 10 disc diameter or more being recognized as high risk of retinal neovascularization development, from previous studies using limited 7-field imaging devices as well as from current studies with advanced UWF-FA. $^{6-9}$ In contrast, published data on ARI are very limited in BRVO, and the studies available had applied ARI of 5 DD or more as the arbitrary guide for NVE risk in ischemic BRVO. ${ }^{1,5}$

To the best of our knowledge, we provide the first ARI analysis in ischemic BRVO eyes using UWF-FA. Our cohort shows that NVE development can arise from 

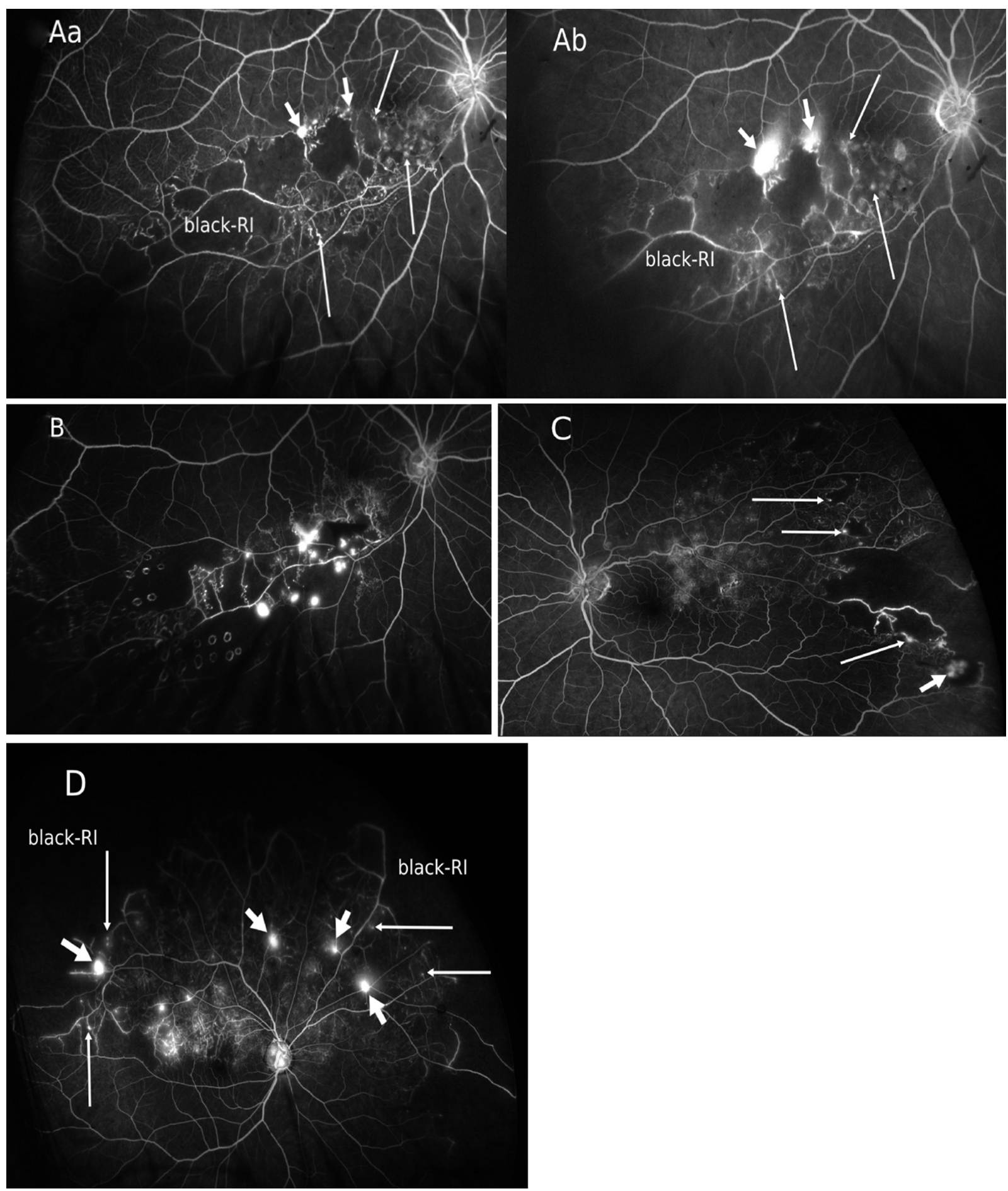

Figure 2 Retinal neovascularizations and angiographic pattern. All the figures (A-D) here are ultra-widefield fluorescein angiograms (UWF-FA) of different patients who had sustained branch retinal vein occlusions. The UWF-FAs depict a recurring theme of angiographic pattern (3Bs pattern); presence of black retinal ischemia (black-RI), budding of retinal microvascular anomalies (RMAs) originating from the same watershed border of black-RI as retinal neovascularizations (NVEs). (A) (a-b) are UWF-FA phases of a patient showing the sectorial black-RI located in the inferotemporal retina involving inferior macula. Active NVEs are seen as hyperfluorescent spots (short arrows) at early phase of angiogram (2Aa) and classically leak fluorescence profusely at the late phase of angiogram (2Ab). The NVEs arise from the water-shed border of black-RI and in this case, are within the macula region. UWF-FA also depicts numerous tiny hyperfluorescent dots of RMAs (long arrows) in early phase of angiogram (2Aa) at the water-shed border but unlike NVEs, they do not leak profusely at late phase (2Ab). (B) UWF-FA of a different patient with branch retinal vein occlusion, showing residual black-RI from insufficient laser treatment hence leading to the proliferation of NVEs with localized pre-retinal hemorrhage in the inferior macula. Note numerous active NVEs arise from the border of the water-shed border, some arise along the venous border within the black-RI. (C) UWF-FA shows patchy ischemic changes in posterior pole but black-RI is most prominent in the far peripheral retina with numerous sprouting NVEs and RMAs (tiny hyperfluorescent dots, long arrows) at the water-shed border. The largest NVE (short arrow) has already bled with localized pre-retinal hemorrhage. (D) UWF-FA of a patient with superior hemi-retinal vein occlusion. The UWF-FA depicts extensive retina ischemia in the superior hemisphere of retina, the black-RI is most prominent at the far peripheral retina. Numerous active NVEs (short arrows) arise from the watershed border and along venous border within the black-RI. RMAs are seen as fainter tiny fluorescein dots (long arrows) scattered along the water-shed border and within the black-RI. 
Table 3 Clinical and Ultra-Widefield Angiographic Findings

\begin{tabular}{|c|c|}
\hline & $\begin{array}{l}\mathbf{N}=60 \\
\text { Eyes }\end{array}$ \\
\hline Presence of angiographic black-RI & \\
\hline $\begin{array}{l}\text { Black-RI involved posterior pole/mid and far peripheral } \\
\text { retina }\end{array}$ & $60(100 \%)$ \\
\hline Black-RI inclusive part of macula & $40(67 \%)$ \\
\hline $\begin{array}{l}\text { Presence of angiographic water-shed ischemic } \\
\text { border }\end{array}$ & $60(100 \%)$ \\
\hline $\begin{array}{l}\text { Presence of RMAs at water-shed ischemic } \\
\text { border }\end{array}$ & $60(100 \%)$ \\
\hline $\begin{array}{l}\text { Presence of NVE network within black retinal } \\
\text { ischemia: }\end{array}$ & \\
\hline Single NVE & $2(3 \%)$ \\
\hline Multiple NVEs & $58(97 \%)$ \\
\hline NVE sites within black retinal ischemia & \\
\hline (a) Arise at water-shed ischemic border & $25(42 \%)$ \\
\hline $\begin{array}{l}\text { (b) Arise at a "vessel-border" within black retinal } \\
\text { ischemia }\end{array}$ & $18(30 \%)$ \\
\hline (c) Arise from both (a) and (b) & $17(28 \%)$ \\
\hline Any part of NVE network within macula region & $31(52 \%)$ \\
\hline Presence of retinal ghost vessels & $47(78 \%)$ \\
\hline Black-RI presence around a ghost vessel & $100 \%$ \\
\hline
\end{tabular}

Abbreviations: $\mathrm{N}$, total number of eyes studied in this cohort; black-RI, black retinal ischemia; RMAs, retinal microvascular anomalies; NVE, retinal neovascularization.

various ARI sizes, ranging from the smallest ARI of 5DD (in only two patients) to the largest 354DD (Table 2). The very large mean ARI of 114DD provides clear evidence of a better mode of imaging, benefiting from a more complete visualization of the whole of ischemic retinal territory, in comparison to the older imaging of 5DD ischemic reference. ${ }^{1}$ We adhered to the ARI being measured against the disc diameter size, as optic disc diameter provides a practical and easily relatable clinical reference in disease management. Although UWF-FA has its limitations in providing high accuracy in ischemia mapping (for examples limited by 2-dimensional digital mapping and view obscuration from eyelashes), it nevertheless allows a significantly improved visualization and easy depiction of the actual size of an ischemic retina in one single-center $200^{\circ}$ wide-view image. Furthermore, UWF-FA enables an easily accessible mapped image, which serves as a useful guide when performing target laser photocoagulation.

\section{Retinal Neovascularization (NVE): Characteristics and Locations}

We have identified that multiple NVE development is a common occurrence in ischemic BRVO eyes with large ARI. There were only two eyes with evidence of a single NVE development in a relatively smaller ARI (each with ARI of 5DD and 12DD). In particular, we recorded NVE networks in all sectors of ischemic retina from the posterior pole to the far-peripheral retina. The ischemic macular area was not spared from NVE development. In the BRVO study by Yasuda et $\mathrm{al}^{5}$ on 40 eyes, NVE was more commonly observed in eyes with larger nonperfused retina in the posterior pole than in mid-periphery, but not observed in the farperipheral retina in their cohort. ${ }^{5}$ However, our observations are consistent with Prasad et $\mathrm{al}^{10}$ in reporting NVE development in untreated or under-treated nonperfusion retina at any location of retina. ${ }^{10}$ In the present cohort, the location of NVE is primarily confined to an area of persistent (or residual) black-RI defined by the initial BRVO occlusion territory boundary, which may or may not affect the macula

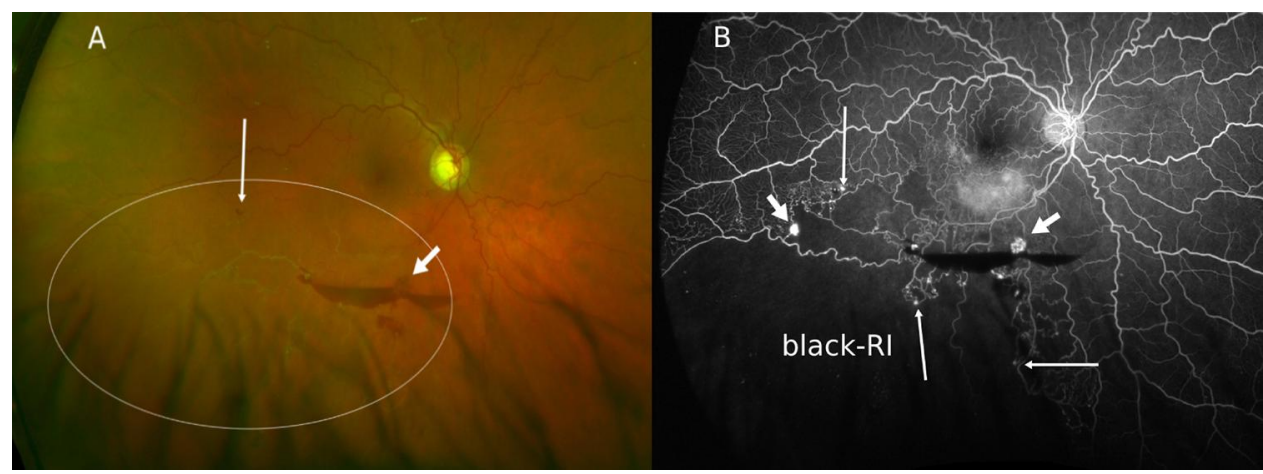

Figure 3 Retinal ghost vessels and retinal microvascular anomalies. (A) An Optos color image showing a visible network of ghost vessels in the inferotemporal retina and a nearby small retinal microvascular anomaly (RMAs, long arrow) indicating an "old" branch retinal vein occlusion (BRVO). Although the patient's vision was not affected, a pre-retinal hemorrhage with a visible retinal neovascularization (NVE, short arrow) suggested progression to late complication from a previous untreated BRVO episode. (B) The Optos ultra-widefield fluorescein angiogram (UWF-FA) of the same eye depicting the presence of black retinal ischemia (black-RI) corresponding to the location of the ghost vessels. In addition to outlining the extent of black-RI, UWF-FA is also distinguishing more NVEs (short arrows) and numerous RMAs (long arrows) along the water-shed border of black-RI in comparison to clinical or photographic detection. 
or the far-peripheral retina. In practical terms, recognizing the presence or persistence of retinal ischemia regardless of its location, may be as important as knowing the size of ARI in assessing the risk of NVE development.

\section{Duration of NVE Development and Progression}

Inadequate laser photocoagulation to the ischemic retina in BRVO can allow the development or progression of NVE. ${ }^{10}$ This is a commonly encountered complication in real world clinical practice, as is also seen in our cohort. At the time of angiographic diagnosis of NVEs, 43\% (26/ 60 eyes) had received prior laser and 33\% (23/60 eyes) received anti-VEGF treatments, the results further questioned the role of short-acting anti-VEGF in ischemic eyes and inadequate laser on ischemic retina allowed the progression of severe complications. ${ }^{1,7}$ Although 12/60 eyes (20\%) of our cohort had angiographic record tracing the NVE development over 7 to 50 months over the follow-up period, this time scale remains an approximation only, as it is impossible to capture the exact time scale of $\mathrm{NVE}$ development in a retrospective study given the data were based on the first re-presentation of NVE bleeding symptoms or when new NVE was first captured by repeated UWF-FA, often at no more than 4-6 monthly intervals. Similarly, 35/60 eyes (58\%) had vitreous hemorrhage as the initial presentation, whilst only $10 / 60$ patients (17\%) were able to clearly remember their previous episode of BRVO diagnosis with or without treatment. Thus, our records would be based on their symptoms rather than the actual NVE development, and it is very likely that NVE development occurs much earlier prior to vitreous hemorrhage. For example, NVE development occurs when residual ischemic retina is left untreated: Figure 4 shows a patient who had evidence of aggressive NVE progression from persisting ischemic retina which received incomplete laser coverage over the 1-2 year period.

In summary, although our cohort shows NVE could arise from any size of black-RI, we could not speculate on the time scale of NVE development. Logistically, a smaller retinal ischemia may take longer to harbor NVE of a smaller scale. With that, it is reasonable to stress the importance in repeating UWF-FA, ensuring laser treatment is effectively delivered to the recognized



Figure 4 Retinal neovascularizations progression from under-treated retinal ischemia. The patient presented with left eye recurrent vitreous hemorrhage following the diagnosis of a supero-temporal branch retinal vein occlusion (BRVO). Ultra-widefield fluorescein angiograms (UWF-FA) were performed at 5 to 9 months interval showing the course of retinal neovascularizations (NVE) progression over the follow-up period whilst receiving laser treatments. (A) Angiographic " 3 Bs pattern" was observed with a small NVE developed within the ischemic macula to account for the initial mild vitreous hemorrhage. Target laser photocoagulation was requested on black retinal ischemia (black-RI). (B) Seven months post-laser treatment, multiple new NVEs were seen at different locations, developed from residual inadequately treated black-RI territory. One NVE was at the water-shed border, one was along a major vessel within the black-RI. Further laser treatment was requested. (C) Five months later, the patient presented with further vitreous hemorrhage due to progression of NVE when residual black-RI remained under-treated. (D) Total resolution of all NVEs confirmed from repeated UWF-FA 9 months after the last laser treatment, showing sufficient laser coverage of the entire black-RI area. The patient had no further vitreous hemorrhage and retained excellent vision despite the disease and treatment proximity to fovea. 
black-RI and lessening the risk of future NVE development.

\section{Novel Angiographic Features: Retinal Microvascular Anomalies (RMAs) and a Common Angiographic Pattern}

Vitreous hemorrhage is a late complication of BRVO from established NVE, and NVE itself only develops at the later stage of the disease where there is persistent retinal ischemia from lack of treatment. In our cohort, we recorded the presence of other clinical features commonly seen in the late phases of BRVO, ghost vessels in 47/60 eyes (78\%) and RMAs in $100 \%$ of the eyes (Table 3 ). Both ghost vessels and RMAs are often regarded as benign features and appear at later stages of BRVO. They are associated with the "healing-phase," after resolution of retinal hemorrhages. However, our angiographic analysis challenges this concept. We observed that both ghost vessels and RMAs are associated with black-RI such that black-RI is consistently a feature around ghost vessels and RMAs are located at the angiographic water-shed ischemic border of the black-RI (100\%). Importantly, of all the NVEs developed within the defined black-RI area, 70\% (42/60 eyes) shared the sites with RMAs at the water-shed ischemic border (Table 3). RMAs are distinguishable from NVEs by means of fluorescein angiography (Table 1 definitions). As both RMA and NVE shared the site of origin, it raises the hypothesis that these RMAs might be the "budding" phase of NVEs, a similar concept to that of intraretinal microvascular anomaly (IRMA) as one of the pre-cursors to NVE in advanced diabetic retinopathy. ${ }^{11}$ The study by Pan et al, ${ }^{11}$ based on both fluorescein angiography and optical coherence tomography angiography, also reported IRMA and NVE originating at the margin of a nonperfused retina. ${ }^{11}$ Another interesting study that supports our hypothesis is from an experimental BRVO animal study, microvascular remodelling upstream of the BRVO occlusion site was recorded, and the formation of RMA was similarly observed at the border of occluded territory. ${ }^{12}$

In summary, we have observed a recurring pattern of three common angiographic features in these ischemic BRVO eyes which had established active NVEs. We refer to these as the angiographic "3Bs" pattern (black-RI, watershed ischemic border, budding of RMA) in our routine clinical management of ischemic BRVO patients (Table 1 definition, Figures 1-4). The acronym provides an easy description of these recurrent angiographic features which may be useful in alerting clinicians to observe potential NVE development and hence to guide efficient therapeutic management. In our experience, patients referred with "old BRVO" with inactive clinical features (such as ghost vessel or RMAs) would benefit from UWF-FA investigation to exclude the presence of retinal ischemia (untreated or under-treated).

\section{Strengths and Limitations}

The strengths of this study include good sample size and core uniform disease representation as well as availability of ultra-widefield imaging on all studied patients.

The availability of UWF-FA has enhanced our understanding of this particular disease subgroup and could be readily applicable to real-world clinical practice when clinicians are investigating these patients using UWF-FA. Our study result also helps practitioners to have a fair clinical estimation of the ischemic retina territory in needing laser coverage for BRVO patients who are unable to have fluorescein angiography.

The limitations of our study include its retrospective and observational design on real-world data and that information regarding the duration of symptoms/disease was obtained through self-reporting rather than by objective medical investigations at planned intervals. There is also a small amount of variability in ARI analysis as this relies on mapping on the ischemic retina visualized on the best UWF-FA image. This method remains the best available technology and facility to date.

In conclusion, we have reported a recognizable recurring angiographic pattern in eyes with established active BRVONVEs using UWF-FA, and that BRVO-NVEs can develop from varied ARI sizes, including eyes with under-treated retinal ischemia. The pathogenesis of NVE development remains complex and these angiographic features may have been simply unrecognized by limitations of previous imaging devices. The utility of UWF-FA has indeed facilitated better clinical management in patients with retinovascular diseases. Our results would hope to stimulate future long-term prospective studies in similar diseases.

\section{Acknowledgments}

We thank our colleagues Vinaya Felicida, Faye Horner, Amoy Ramsay and Eesha Gokhale for finalizing the acronym (3Bs pattern) and their dedication in RVO clinical services.

\section{Funding}

There is no funding to report. 


\section{Disclosure}

$\mathrm{HK}$ has received travel reimbursements from Bayer and Novartis. The authors report no other conflicts of interest in this work.

\section{References}

1. Branch Vein Occlusion Study Group. Argon laser scatter photocoagulation for prevention of neovascularization and vitreous haemorrhage in branch vein occlusion: a randomized clinical trial. Arch Ophthalmol. 1986;104(1):34-41. doi:10.1001/archopht.1986.01050130044017

2. Ferrara N. Vascular endothelial growth factor: basic science and clinical progress. Endocr Rev. 2004;25(4):581-611.

3. Gozawa M, Takamura Y, Miyake S, et al. Photocoagulation of the retinal nonperfusion area prevents the expression of the vascular endothelial growth factor in an animal model. Invest Ophthalmol Vis Sci. 2017;58(13):5946-5953. doi:10.1167/iovs.17-22739

4. Patel SN, Shi A, Wibbelsman TD, Klufas MA. Ultra-widefield retinal imaging: an update on recent advances. Ther Adv Ophthalmol. 2020;12:2515841419899495.

5. Yasuda Y, Hirano Y, Esaki Y, et al. Peripheral microvascular abnormalities detected by wide-field fluorescein angiography in eyes with branch retinal vein occlusion. Ophthalmic Res. 2019;61(2):107-114. doi:10.1159/000488496
6. Tsui I, Kaines A, Havunjian MA, et al. Ischaemic index and neovascularization in central retinal vein occlusion. Retina. 2011;31 (1):105-110. doi:10.1097/IAE.0b013e3181e36c6d

7. Wykoff C, Brown D, Croft D, Jr MJ, Wong T. Progressive retinal nonperfusion in ischaemic central retinal vein occlusion. Retina. 2015;35(1):43-47. doi:10.1097/IAE.0000000000000277

8. Nicholson L, Vazquez-Alfageme C, Patrao NV, et al. Retinal nonperfusion in the posterior pole is associated with increased risk of neovascularization in central retinal vein occlusion. $\mathrm{Am}$ J Ophthalmol. 2017;182:118-125. doi:10.1016/j.ajo.2017.07.015

9. Central Vein Occlusion Study Group. A randomized clinical trial of early panretinal photocoagulation for ischemic central vein occlusion. The central vein occlusion study Group N report. Ophthalmology. 1995;102(10):1434-1444. doi:10.1016/S0161-6420(95)30848-2

10. Prasad P, Oliver S, Coffee R, Hubschman JP, Schwartz S. Ultra-widefield angiographic characteristics of branch retinal and hemicentral retinal vein occlusion. Ophthalmology. 2010;117(4):780-784. doi:10.1016/j.ophtha.2009.09.019

11. Pan J, Chen D, Yang X, et al. Characteristics of neovascularization in early stages of proliferative diabetic retinopathy by optical coherence tomography angiography. Am J Ophthalmol. 2018;192:146-156. doi:10.1016/j.ajo.2018.05.018

12. Genevois O, Pâques M, Simonutti M, et al. Microvascular remodeling after occlusion-recanalization of a branch retinal vein in rats. Invest Ophthalmol Vis Sci. 2004;45(2):594-600. doi:10.1167/ iovs.03-0764
Clinical Ophthalmology

\section{Publish your work in this journal}

Clinical Ophthalmology is an international, peer-reviewed journal covering all subspecialties within ophthalmology. Key topics include: Optometry; Visual science; Pharmacology and drug therapy in eye diseases; Basic Sciences; Primary and Secondary eye care; Patient Safety and Quality of Care Improvements. This journal is indexed on PubMed

\section{Dovepress}

Central and CAS, and is the official journal of The Society of Clinical Ophthalmology (SCO). The manuscript management system is completely online and includes a very quick and fair peer-review system, which is all easy to use. Visit http://www.dovepress.com/ testimonials.php to read real quotes from published authors. 